引用格式: 许恩银, 陶韵, 杨红强. LULUCF 关联林业碳问题研究进展 [J]. 资源科学, 2019, 41(9): 1641-1654. [Xu E Y, Tao Y, Yang H Q. Research progress of forestry carbon issues related to land use, land-use change and forestry[J]. Resources Science, 2019, 41(9): 1641-1654.] DOI: 10.18402/resci.2019.09.06

\title{
LULUCF 关联林业碳问题研究进展
}

\author{
许恩银 ${ }^{1,2}$, 陶 韵 1,2 , 杨红强 $1,2,3$
}

（1. 南京林业大学经济管理学院，南京 210037;2. 国家林业局林产品经济贸易研究中心， 南京 210037;3. 南京大学长江三角洲经济社会发展研究中心, 南京 210093)

\begin{abstract}
摘 要: 土地利用、土地利用变化和林业(LULUCF) 关联的碳问题是影响适应和减缓气候变化的重要问题。 厘清近 20 年来 LULUCF 涉及林业碳科学的国际研究进展, 对作好响应气候变化的林业政策制定及调整具有重要 意义。本文应用 CiteSpace 分析工具, 以 Web of Science 数据库 1999-2018年的 175 篇文献为研究对象, 运用知识 图谱分析, 归纳了LULUCF 林业碳问题的研究趋势。结果表明: 1 “制度和方法的改进” “林业管理行为”和“环境目 标” 3 大类群是当前LULUCF 的研究主题。(2)德国、意大利、英国和美国是研究 LULUCF 的主要国家, 分别侧重于 研究毁林、林业项目、经济成本问题和部门响应机制。(3LULUCF 社会科学的研究重点是土地利用管理、政策制度 的响应机制和减缓气候变化的信贷等经济调节杜杆问题,主要方法是以情景分析法和一般均衡模型为代表的工程 管理学与经济学方法。本文对于中国 LULUCF 未来的研究提供了相关建议: 完善LULUCF碳核算基础方法和土地 利用及动态变化监测的理论及手段, 重视林业管理项目的科学评估及其对林业政策调整的指导作用, 加快推进LU-

LUCF 碳预算及土地利用部门针对减排方案的制订。
\end{abstract}

关键词:LULUCF;CiteSpace; 林业碳; 聚类;林业管理项目; 碳预算; 碳抵消

DOI :10.18402/resci.2019.09.06

\section{1 引言}

人类活动所引起的土地利用变化是导致温室 气体浓度上升和气候变化的重要原因之一, 国际上 关于碳汇碳源的研究中, 土地利用变化与生态系统 涉及的碳问题是应对气候变化的重点 ${ }^{[1]}$ 。《联合国气 候变化框架公约》(United Nations Framework Convention on Climate Change, UNFCCC) 中《京都议定 书》(Kyoto Protocol, KP) 第二承诺期的焦点问题在 于资金机制安排、技术转让、发达国家履约、土地利 用和林业, 其中林业议题的重点是 “土地利用、土地 利用变化和林业” (Land Use, Land Use Change and Forestry, LULUCF)、“减少发展中国家毁林和森林
退化所致碳排放” (Reducing Emissions from Deforestation and Forest Degradation, REDD+) 和“木质林 产品” (Harvested Wood Products, HWP) 3 个方面 ${ }^{[2]}$ 。 UNFCCC 三大林业核心议题 LULUCF、REDD+和 HWP 涉及的气候变化及碳问题侧重点见图 1。

LULUCF 是指由于人为直接利用土地、改变土 地利用和林业活动而产生的温室气体的排放和清 除 ${ }^{[3]}$ 。1 995 年联合国政府间气候变化专门委员会 (Intergovernmental Panel on Climate Change, IPCC) 第二次评估报告 ${ }^{[4]}$ 首次将林木种类构成发生变化的 生态系统的过程和向大气中释放碳结合起来, 这是 LULUCF 的雉形; 1998 年科技咨询机构 (Subsidiary

收稿日期: 2019-04-02, 修订日期: 2019-07-12

基金项目: 江苏省“333 高层次人才工程”科研项目(BRA2018070); 国家社会科学基金重点项目(14AJY014); 江苏省研究生科研与实践创新计 划项目(KYCX18_0974)。

作者简介: 许恩银,女, 江苏南通人,博士生, 主要研究方向为土地利用、土地利用变化与林业。E-mail: xuenyin068@163.com 通讯作者: 杨红强, 男, 陕西渭南人, 博士, 教授, 博导, 主要研究方向为气候变化及资源利用, 林产品贸易与环境。E-mail: yhqnfu@aliyun.com 


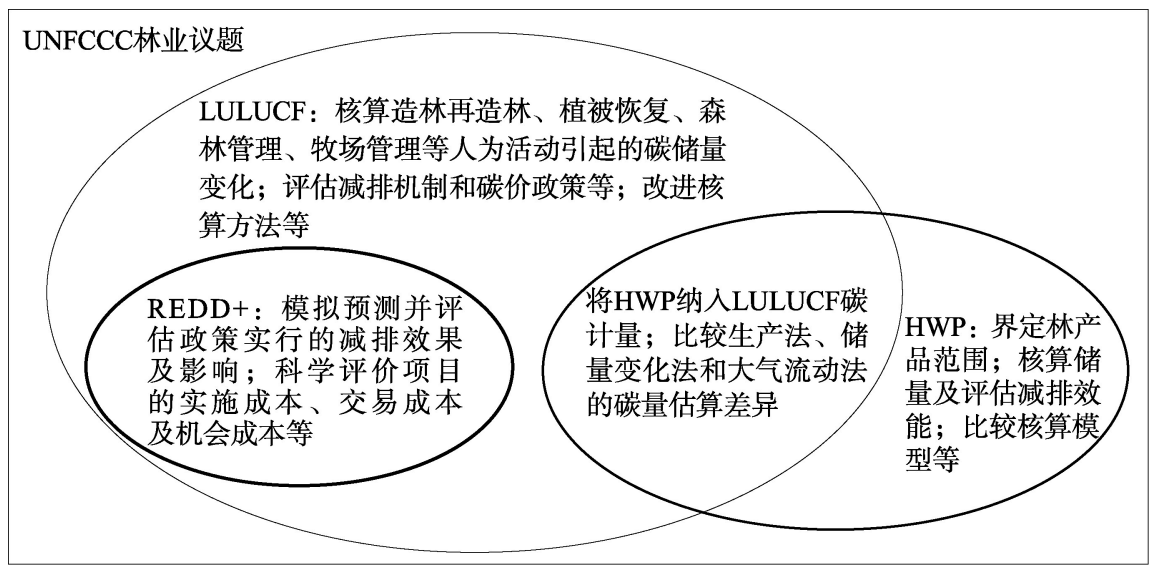

图 1 UNFCCC 林业核心议题及碳问题

Figure 1 The core forestry and carbon issues in the United Nations Framework Convention on Climate Change (UNFCCC)

Body for Scientific and Technological Advice, SBSTA) 提出建议审查与“土地利用、土地利用变化和 林业活动”活动有关的碳固存战略以及 KP 相关条 款的科学和技术理解状况的报告, 同年 IPCC 第 14 次会议批准了特别报告的范围、结构和大纲;2000 年关于 “土地利用、土地利用变化和林业的特别报 告” (IPCC Special Report on Land use, Land- Use Change and Forestry, SR-LULUCF) 正式发布;2001 年 IPCC 第三次评估报告 ${ }^{[5]}$ 中第一次正式出现 “LULUCF”, 并且第三工作组报告具体分析它的减排技 术、发展障碍、发展机会和对土地利用的影响; IPCC 第四次评估报告 ${ }^{[6}$ 将 LULUCF 与能源供应、交通运 输、工业、农业等行业并列作为鼓励采取减缓行动 的关键行业; IPCC 第五次评估报告 ${ }^{[7]}$ 将 LULUCF 作 为影响适应和减缓气候变化及其共生效益联系的 主要因素之一。

2000 年后, 国际范围 LULUCF 项目的碳减排潜 力、社会总成本及其对环境和农村生计的影响是主 要关注问题 ${ }^{[8-10]}$ 。至 2010 年, LULUCF 又被作为温 室气体清单核算的重要部门之一, 核算方法的适用 性和不确定性程度颇受关注 ${ }^{[11-13]}$ 。当前, 对 LULUCF 的研究集中于核算 LULUCF 缓解温室气体排 放的潜力, 评估其为实现减排目标做出的贡献 ${ }^{[14]}$, LULUCF 的巨大缓解潜力让清洁发展机制 (Clean Development Mechanism, CDM) 和其他气候政策工 具都有望为其提供资金支持 ${ }^{[15]}$ 。LULUCF 涉及碳储 量的变化受许多因素的影响,包括不同土地用途的
适用性及碳固存、碳价格政策的有效性等 ${ }^{[16,17]}$, 同时 一系列评估土地用途适用性和政策有效性的方法 也得到了关注 ${ }^{[18]}$ 。中国作为碳排放大国,近十多年 来, 关于 LULUCF 的研究以张小全 ${ }^{[1,19-21]}$ 、李玉娥 ${ }^{[22-24]}$ 、 刘硕 ${ }^{[22,23]}$ 、赵志平 ${ }^{[25]}$ 和李智勇 ${ }^{[26]}$ 等学者为代表, 主要 涉及国际气候谈判责任、 $K P$ 承诺期减排目标、土地 利用变化与生态系统碳库、LULUCF 温室气体清单 编制、LULUCF 碳汇计量监测体系等方面。具体关 联到林业及其气候变化, 国内研究重点在生物量、 碳储量、HWP、森林管理和森林碳汇等方面。国内 的研究,一方面考虑了 LULUCF 在完成 KP 减排目 标中的作用及对碳排放交易市场的影响,另一方面 侧重不同 LULUCF 活动形式及碳计量方法在中国 的适应性及改进问题,既有的研究为以森林管理碳 汇和 HWP 碳储量为代表的林业碳科学的进一步发 展提供了重要基础。自 20 世纪 90 年代以来, 中国 一直在实施植树造林和森林保护计划, 代表性重大 林业管理项目诸如退耕还林、天然林保护工程等 ${ }^{[27]}$, 这些重大的林业管理项目引致的 LULUCF 问题直 接关系中国林业在应对气候变化的能力, 准确把握 LULUCF 林业碳问题的国际研究趋势及方法学, 对 科学评估中国重大林业管理项目的碳贡献及政策 调整具有重要参考价值。

本文目的在于梳理近 20 年 LULUCF 领域涉及 林业碳科学问题的文献,分析林业管理行为及碳活 动国际间的研究重点及评估方法, 比较国内与其他 国家的研究差异,归纳 LULUCF 林业碳问题的研究 
趋势及中国应注意的问题。本文利用CiteSpace 5.3. R8工具对目标领域的被引文献进行描述统计和数 据挖掘, 通过知识图谱和高中心性、高被引文献, 归 纳提炼 LULUCF 林业碳问题研究重点。LULUCF 发展的不同阶段在 IPCC 报告使用的表达方式不一 致, 因此收集文献时检索条件主题词包含了“土地 利用、土地利用变化和林业” “土地利用变化和林 业”和“林业和其他行业土地利用” 3 种表达术语。 本文中将碳排放、碳吸收和碳储存等统称为碳活动。

\section{2 数据来源与研究方法}

\section{1 数据来源}

本文以 Web of Science (WOS) 核心合集数据库 作为数据来源。检索主题为 “ 'LULUCF” OR 'Land Use, Land- Use Change and Forestry' OR 'LUCF' OR 'Land-Use Change and Forestry' OR 'FOLU' OR 'Forestry and Other Land Use' ”; 检索 时间跨度为 1999-2018 年; 文献类型包括文章、综 述、会议摘要和期刊评论等,共检索到 283 篇文献。

本文研究步骤分 2 个部分。其一, 通过分阶段 的关键词共现图谱分析 LULUCF 林业碳问题的研 究历史演进, 再通过文献共被引网络和国家合作网 络分析其关联的代表文献和聚类。在这 283 篇文献 中, $54.10 \%$ 的文献与环境生态学有关, $19.10 \%$ 的文 献与林业有关, $9.50 \%$ 的文献与农业有关, 其他还涉 及了地质学、能源燃料、生物多样性保护和水资源 等专业。为了使林业碳的研究角度更精确, 在 283 篇文献中加人检索词“forestry” 和“carbon”, 精炼出 与林业和碳问题有关的 175 篇文献作为本文的样本。

其二, 考虑到在聚类结果中自然科学类文献较 多, 社会科学类文献在图谱中不明显, 而 LULUCF 林业碳问题与社会经济因素关联性强, 其管理方式 也关系到减排措施的实施效果。因此, 在这 175 篇 文献中加人检索条件““management” or 'social” or “economic'”, 精炼出涉及社会经济因素的 83 篇文 献, 对这 83 篇文献进行关键词共现分析, 进一步探 究LULUCF林业碳问题在社会科学上的研究进展。

\section{2 研究方法}

\subsubsection{CiteSpace 聚类原理及逻辑}

为合理评价 LULUCF 国际文献的关联性及研 究趋势, 本文引人文献关联度评价的主流方法
CiteSpace ${ }^{[28,29]}$ 进行知识图谱聚类和归纳分析。 CiteSpace 的核心功能是分析学科研究前沿与其知 识基础之间的关系,继而提供针对研究对象的历时 性变化趋势。CiteSpace使用 3 个基本概念对其工作 原理进行阐释, 分别是 “研究领域” “研究前沿” 和 “知识基础”。

CiteSpace 对这 3 个基本概念的逻辑关系定义为 $\Phi(t): \Psi(t) \rightarrow \Omega(t)$ 。其中, $\Psi(t)$ 表示某研究领域的 “研究前沿”, 它由一组在 $t$ 时刻与研究前沿新趋势 密切相关的专业术语或短语组成; $\Omega(t)$ 代表该研究 领域的“知识基础”,它主要由包含前沿术语的研究 论文所引用的大量参考文献组成; “研究领域” $\Phi(t)$ 则被概念化为一个从“研究前沿” 到“知识基础”的 时间映射。对上述基本概念及其关系的一个更为 形式化的描述如下:

$$
\begin{aligned}
& \Psi(t)=\left\{\text { term } \mid \text { term } \in S_{\mathrm{TI}} \cup S_{\mathrm{AB}} \cup S_{\mathrm{DE}} \cup S_{\mathrm{ID}}\right. \\
& \wedge \text { IsHotTopic }(\text { term, } t)\} \\
& \Omega(t)=\left\{\text { article } \mid \text { term } \in \Psi(t) \cap \text { term }_{\in} \text { article }_{0} \cap\right. \\
& \text { article }_{0} \rightarrow \text { article }_{\}}
\end{aligned}
$$

式中: $S_{\mathrm{TI}} 、 S_{\mathrm{AB}} 、 S_{\mathrm{DE}}$ 和 $S_{\mathrm{ID}}$ 分别表示来自论文题目、 摘要、作者关键词和增补关键词字段的一系列专业 术语; IsHotTopic (term, $t)$ 是一个布尔函数, 表示 $t$ 时刻 term 是否为一个热点术语; 而 article $_{0} \rightarrow$ article 表示论文 article $_{0}$ 引用了论文 article ${ }^{[30]}$ 。具体来说, CiteSpace利用自动聚类标签视图从时间、分类不同 角度展示出研究领域的分布情况,通过谱聚类算法 生成知识聚类,如文献共被引网络和作者共被引网 络等,再通过施引文献提取标签词, 用来对应知识 基础的研究前沿 ${ }^{[31]}$ 。

\subsubsection{CiteSpace 评价指标}

CiteSpace 中知识图谱和节点各自有不同的评 价指标, 知识图谱评价指标越高, 则绘制效果越显 著,节点的评价指标越高,则更具代表性。

知识图谱依据网络结构和聚类的清晰度提供 了模块值 (Modularity, $Q$ 值) 和平均轮廓值 (Silhouette, $S$ 值) 2 个指标, 作为评判图谱绘制效果的依 据。(1)模块值表示聚类结构的效果, 计算公式为 $Q=\sum_{i}\left(e_{i i}-a_{i}^{2}\right)$, 其中 $i$ 是划分好的聚类编号, $e_{i i}$ 是 聚类内部连线占全图所有连线的比例, $a_{i}$ 是聚类 $i$ 
相关的连线占全图所有连线的比例。聚类效果越 好, 内部连线就越多, 从而 $e_{i i}$ 越大, $\mathrm{Q}$ 值越大, 一般 $Q>0.30$ 就认为聚类结构显著 ${ }^{[31]}$ 。2)平均轮廓值表 示聚类的同质性, 即是否具有足够的相似性。单个 样本点的轮廓系数是 $S_{i}=1-a / b, a$ 为点 $i$ 与所在 类中其他点的平均距离, $b$ 为点 $i$ 与最接近点 $i$ 所在 类的类中各点的平均距离。平均轮廓值是各样本 点轮廓值的平均数,一般认为 $S>0.50$ 即聚类合理, $S>0.70$ 即聚类是令人信服的 ${ }^{[31]}$ 。

节点的中心性和被引频次分别表示节点信息 在结构和基础上所起的作用, 高中心性和高被引频 次的节点信息在结构和基础上占据重要位置, 可以 作为某个阶段的研究重点代表。(1)节点中心性 (Centrality) 即网络中通过某节点的任意最短路径 的条数, 是网络中节点在整体网络中所起连接作用 大小的度量。中心性不低于 0.10 的节点称为关键 节点, 关键节点控制科研走向, 其他节点以关键节 点为核心构成研究结构。(2)被引频次 (Citation Counts) 是节点信息被样本中其他文献引用的次数, 新兴的研究趋势和新出现的科研主题映射于对应 的高被引节点信息中。

聚类视图(图谱)可表达研究前沿和知识基础、 研究前沿的演变, 以及在演变过程中涉及的关键文 献, 但图谱只能勾勒整个研究领域的概况, 却无法 提供更深人的文献细节信息。首先, 本文依次对 175 篇 LULUCF 林业碳问题的文献进行分阶段关键 词共现、文献共被引和国家合作进行分析; 其次, 提 炼了其中 83 篇与社会科学相关的文献, 并进行了关 键词共现比较; 然后, 将国内研究和其他国家研究 进行对比, 梳理国内研究尚存在的问题; 最后, 结合 对高被引频次、高中心性文献的研读和综合判断， 对该领域国际间的研究趋势及中国相关研究值得 关注的问题提出建议。

\section{3 分类结果及解释}

\subsection{LULUCF 林业碳问题研究的历史演进}

以 1999-2018 年的 175 篇文献为样本, 每 5 年 进行一次关键词共现分析, 文献时间分区设置为 1 年, 节点类型设置为 “Keyword”, 阈值选择设置为 50 。CiteSpace工具依据公式 (1)、(2) 原理, $\Omega(t)$ 为 从 WOS 数据库的各阶段文献, $\Psi(t)$ 为在不同时刻
与 LULUCF 研究热点问题密切相关的术语, 通过时 间映射得到高频次关键词 $\Phi(t)$ ，具体结果在关键词 共现图谱中显示。各图模块值均高于 0.40 , 平均轮 廓值均高于 0.50 , 图谱绘制效果均显著。

本文将相关文献按 5 年分为 4 个阶段 (图 2 ), 历 史演进的聚类结果表明,LULUCF 林业碳问题的研 究在各个阶段呈现递进深人的研究趋势。第一阶 段(1999-2003 年),国际上关于LULUCF 林业碳问 题的研究处于起步阶段,主要关注的是碳固存和成 本问题,合理利用 CDM 达到减排目标是最初的核 心目标。第二阶段 (2004-2008 年),随着 KP 的正 式生效, LULUCF 活动和减排目标成为重点讨论话 题,重点结合 $\mathrm{KP}$ 和马拉喀什协议的规定, 对实现环 境保护的各项 LULUCF 活动进行研究,此阶段的关 键词涉及各部门应对机制、活动对农业的影响、对 土壤碳及大气碳的影响等。第三阶段 (2009-2013 年),此阶段除了碳固存、减排和气候变化等, 发展 中国家和管理问题也成为了热门关键词,这意味着 发展中国家在减排方面的地位提升,森林管理在减 缓气候变化方面的贡献开始受到重视。第四阶段 （2014-2018 年）,2014年后,LULUCF 林业碳问题 的研究内容逐渐丰富,在会议报告方面包括对 KP 和巴黎协定等的分析和评价; 在林业项目方面包括 对毁林、REDD+和森林管理等的模拟预测及评估; 在碳的研究方面包括温室气体排放、土壤有机碳和 碳储存核算等; 在土地利用方面包括农业及农地林 地转化导致的碳储量变化等; 在生态学方面包括生 物量核算、生物多样性保护和替代能源的研究利 用等。

\section{2 聚类标签的分类结果及代表文献}

以 175 篇文献为样本, 文献时间分区设置为 1 年, 节点类型设置为 “Reference”, 阈值选择设置为 50 ,即提取每年被引频次排名前 50 的被引用文献来 生成最终的网络。调整优化后的文献共被引图谱 共识别出 6 个共被引聚类, 分别为多源比较 (Multisource Comparison)、林业发展项目 (Forestry Development Program）、其他土地利用（Other Land Use）、 十大短期部门基准 (Ten Key Short- term Sectoral Benchmark）、信贷核算 (Credible Accounting)和碳 (Carbon)。 
Time span: 1999-2003 (Slice Length=1)

Selection Criteria: Top 50 per slice, $L R F=2, L B Y=8, e=2.0$

Network: $N=2, E=1$ (Density=1)

Pruning: None

Modularity $Q=0.5$

Mean Silhouette $=5$

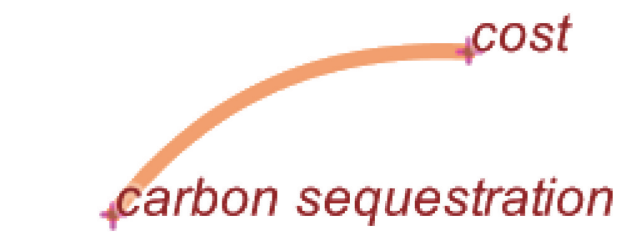

Time span: 2009-2013 (Slice Length $=1$ )

Selection Criteria: Top 50 per slice, $\mathrm{LRF}=2, \mathrm{LBY}=8, \mathrm{e}=2.0$

Network: $N=35, E=99$ (Density $=0.1664$ )

Pruning: None

Modularity $Q=0.4154$

Mean Silhouette $=0.5749$

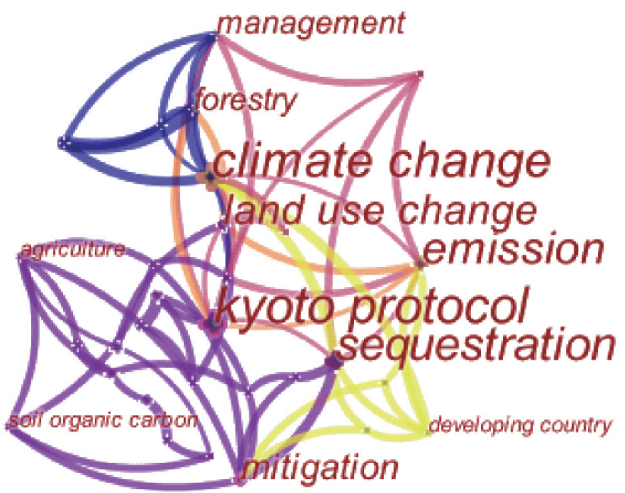

Time span: 2004-2008 (Slice Length=1)

Selection Criteria: Top 50 per slice, $\mathrm{LRF}=2, \mathrm{LBY}=8, \mathrm{e}=2.0$

Network: $N=24, E=46$ (Density $=0.1667$ )

Pruning: None

Modularity $Q=0.591$

Mean Silhouette $=0.878$

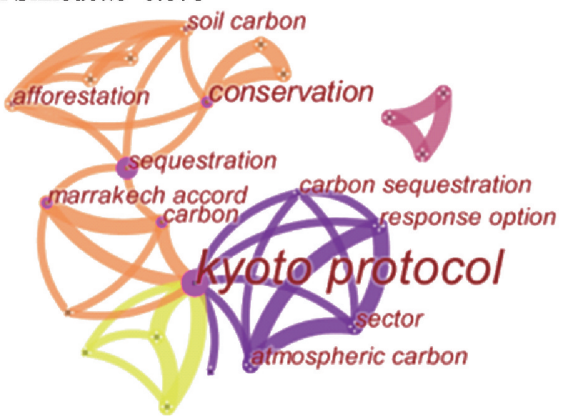

Time span: 2014-2018 (Slice Length=1)

Selection Criteria: Top 50 per slice, $\mathrm{LRF}=2, \mathrm{LBY}=8, \mathrm{e}=2.0$

Network: $N=34, E=92$ (Density $=0.164$ )

Pruning: None

Modularity $Q=0.5027$

Mean Silhouette $=0.7398$

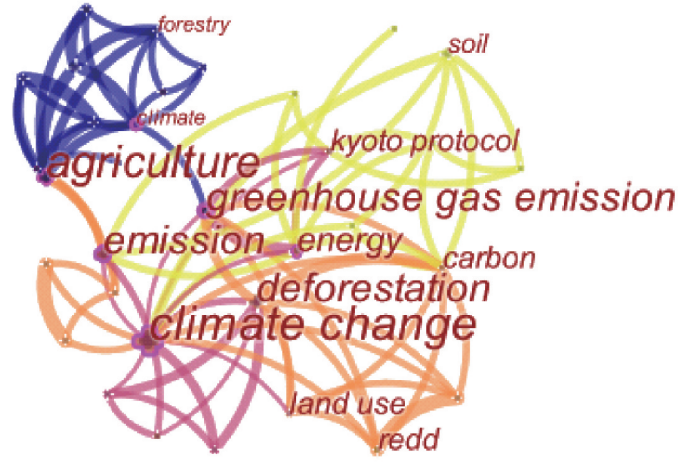

图 2 1999-2018 年 LULUCF 林业碳问题关键词共现图谱

Figure 2 Research clusters and representative literature on forestry carbon issues related to LULUCF, 1999-2018

注: a. 图谱左上角参数涵义:Time span 表示分析的时间区间,括号中为时间切片; Selection Criteria表示阈值选择, Top $n$ per slice 即提取每 个时间切片排名前 $n$ 位的数据来生成最终的网络; $N$ 表示网络的节点数量, $E$ 表示连线数量, Density 表示网络的密度; Pruning 表示网络裁剪的 方法; Modularity $Q$ 表示网络的模块度, $Q>0.30$ 即聚类结构显著; Mean Silhouette表示网络同质性, $S>0.50$ 即聚类合理。b.网络图中节点表示关 键词; 节点的大小与出现频次成正比; 紫色标注的节点表示中心性不低于 0.10 的关键词; 连线的粗细表示关键词之间的联系程度; 连线的颜色 表示共现关系第一次发生的年份, 由蓝到红再到黄色渐变即 1999-2018年随年份递增。

根据研究内容将 6 个聚类标签归为“制度和方 法的改进” “林业管理行为”和“环境目标” 3 个研究 类群,表 1 概述了中心性不低于 0.10 及被引频次高 于 4 的 27 篇文献的主要结论及方法。第一, “制度 和方法的改进” 类群, 包括多源比较和碳聚类, 主要 通过簿记模型 (Bookkeeping Model)、卫星探测数 据、路线图、GIS 和全球生物圈管理模式 (Global Biosphere Management Model, GLOBIOM) 等方法,
估计研究区域的碳储量和碳排放量等, 从而对热带 地区碳密度图和碳储量图等作出补充, 对碳预算和 碳抵消等机制提出建议。第二,“林业管理行为”类 群,包括林业发展项目和其他土地利用聚类,通过 元分析（Meta-Analysis）、全球环境综合评估模型 (Integrated Model to Assess the Global Environment, IMAGE) 和农产品和贸易政策分析国际模型 (International Model for Policy Analysis of Agricultural 


\section{表 1 LULUCF 林业碳问题的研究聚类及代表文献}

Table 1 Research clusters and representative literature on forestry carbon issues related to LULUCF

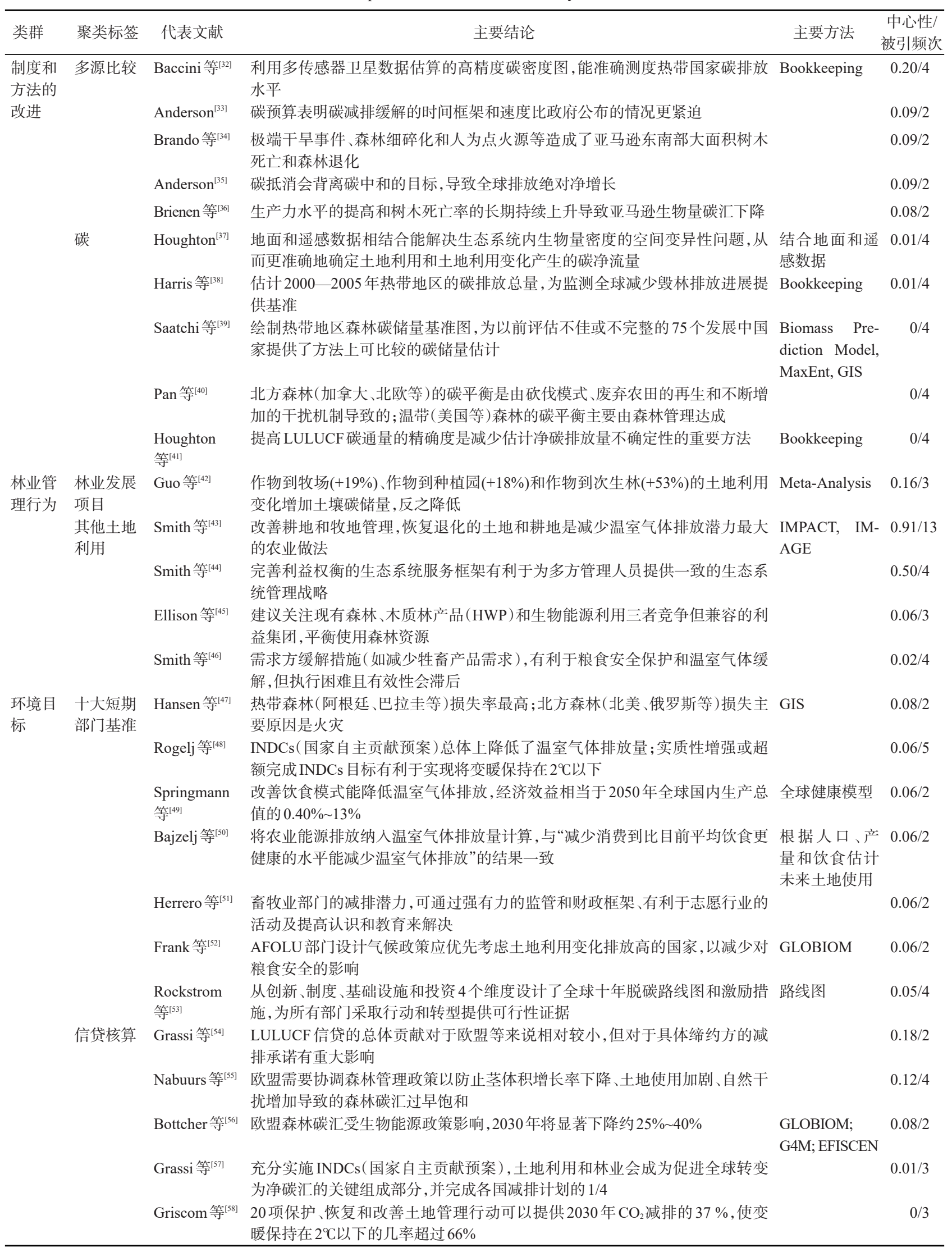


Commodities and Trade, IMPACT) 等方法, 评估恢复 退化土地、再造林和减少产品需求等管理行为对缓 解温室气体排放的影响, 为保护森林碳库和增加碳 储量提出建议。第三, “环境目标”类群,包括十大 短期部门基准和信贷核算聚类,主要以保护环境为 最终目标, 评估部门减排路径和国家自主贡献预案 (Intended Nationally Determined Contributions, IN$\mathrm{DCs}$ )等政策的减排潜力和对环境的影响, 并提出改 善措施。十大短期部门是指 IPCC 评估报告中的能 源供应,工业,交通,建筑,农业、林业和其他土地利 用 (Agriculture, Forestry and Other Land Use, AFOLU)等部门。

\section{3 不同国家研究侧重点}

为了厘清近 20 年来不同国家对 LULUCF 林业 碳问题的研究侧重点, 本节以 175 篇文献为样本进 行分析, 具体操作为文献时间分区设置为 1 年, 节点 类型设置为 “Country”, 阈值选择设置为 50 。根据国 家合作网络得到文献国家分布表 (表2), 德国、意大 利、英国和美国是 LULUCF 研究的重要国家, 德国、 意大利和美国作为中心性最高的 3 个国家, 其研究 会影响其他国家的研究方向和重视程度。澳大利 亚、荷兰、瑞典、芬兰和奥地利等国家在这方面的研 究也较为出色。

各国以缓解气候变化为最终目的, 从碳固存、 碳排放、生物多样性、毁林造林和管理等方面进行 研究并提出改善措施,但是主要关注点各有侧重。 德国重点关注毁林对 AFOLU 部门碳排放的影响和 模型在评估排放模式中的应用。意大利的研究关 注点较多,其中包括森林管理参考水平下碳汇核算
和碳固存和储存项目的分析比较。英国特别关注 土地利用变化对土壤有机碳储量和温室气体排放 的影响, 以及不同政策选择的成本预算和经济效 益。美国的研究问题涉及到比较林业项目在不同 减排机制中的作用, 比较不同部门的温室气体排放 量。荷兰特别关注减排机制对社区林业的影响,主 要目的是期望通过土地管理和林地管理提高储存 碳潜力,并保护森林和生物圈。澳大利亚和瑞典是 研究生物量和生物多样性的重要国家,主要目的是 鼓励协调减少排放量和保护生物多样性的关系。 芬兰和奥地利更关注对森林树种的研究,其中,芬 兰结合松树和云杉等树种生长特点估计欧洲森林 的温室气体排放量和吸收量。上述结果可帮助学 界更便捷地选择、查阅和收集这一领域的主要研究 问题, 及时了解该领域的前沿动态。

\section{4 社科类共现关键词}

为了使社会科学类文献在图谱分析中更具体, 本节以 1999-2018 年的 83 篇与社会经济和管理有 关的文献为样本, 文献时间分区设置为 1 年, 节点类 型设置为 “Keywords”, 國值选择设置为 50 , 得到关 键词共现图谱,关键词分类和具体信息如表 3 所示。

通过关键词共现图谱和节点所对应的文献发 现,LULUCF 林业碳问题在社会科学领域的关键词 大致可以分为 3 类。第一大类是与管理有关的内 容,研究不同实施主体和实施对象的管理模式,包 括分析管理模式的可行性和影响,预测未来发展和 潜在不确定性的程度 ${ }^{[59]}$, 关键词中有“系统”和“影 响”等。第二大类与政策制度相关,一方面分析评 价针对 LULUCF 制定的政策制度并追踪实施进展

表 2 1999-2018 年不同国家对于 LULUCF 林业碳问题研究的侧重点

Table 2 Research focus of different countries on forestry carbon issues related to LULUCF, 1999-2018

\begin{tabular}{clccll}
\hline 序号 & 国家 & 文献数量/篇 & 百分比/\% & 共性研究问题 & \multicolumn{1}{c}{ 侧重研究关键词 } \\
\hline 1 & 德国 & 21 & 12.00 & 碳固存 & 毁林,亚马逊流域,模型,马拉喀什协议,等 \\
2 & 意大利 & 19 & 10.86 & 碳排放 & 毁林,造林,土地退化,等 \\
3 & 英国 & 19 & 10.86 & 气候变化缓解 & 预算,成本,碳汇,土壤有机碳,等 \\
4 & 美国 & 18 & 10.29 & 管理 & 部门,预算,亚马逊流域,响应机制, 等 \\
5 & 荷兰 & 12 & 6.86 & & 欧洲森林,生物量,社区林业,等 \\
6 & 澳大利亚 & 12 & 6.86 & & 生物多样性,保护,造林,等 \\
7 & 瑞典 & 10 & 5.71 & & 生物多样性,土壤有机碳,等 \\
8 & 芬兰 & 9 & 5.14 & & 欧洲森林,苏格兰松树,挪威云杉,等 \\
9 & 奥地利 & 8 & 4.57 & & 森林碳汇,等 \\
\hline
\end{tabular}


情况, 另一方面评估其他领域政策制度的实施对 LULUCF 的潜在影响, 其中出现频次较高的关键词 有“KP”“毁林” “巴黎协定”和“CDM”等。第三大类 偏向于经济环保类, 主要研究减缓措施的经济效益 和碳市场的发展对土地利用变化的影响 ${ }^{[18,46,60]}$, 目的 在于权衡政策选择和经济效益之间的关系, 关键词 以“保护” “成本” 和“减缓”等为代表。LULUCF 林 业碳问题在社会科学领域使用的方法大致分为工 程管理学方法和经济学方法, 其中工程管理学方法 包括情景分析法、马尔科夫链模型、层次分析法、比
较分析法和实地研究法等, 经济学方法主要涉及一 般均衡模型和空间成本效益分析法等。

\section{5 国内与其他国家研究的对比}

表 4 归纳了 1999-2018 年发表 LULUCF 林业 碳问题研究具有代表性的国内外期刊, 另外也对涉 及到中国问题的外文期刊做出统计。其中, Climatic Change, Environmental Science \& Policy 和 Forest Ecology \& Management 期刊发表文献数量较多。结 合期刊的所属领域和 JCR 分区可以发现, 以上期刊 均为环境生态学和林业领域类期刊。国内文献发

\section{表 3 LULUCF 林业碳问题社科研究关键词分类}

Table 3 Classification of social sciences' keywords on forestry carbon issues related to LULUCF

\begin{tabular}{|c|c|c|c|}
\hline 研究类群 & 关键词 & 总频次 & 主要方法 \\
\hline 管理类 & 气候变化, AFOLU, 土地利用,农业, 林业, 管理, 系统 & 39 & $\begin{array}{l}\text { 实地研究法, 评价指标体系 }{ }^{[59]} \text {, 情景分析法, 马尔科夫 } \\
\text { 链模型 }\end{array}$ \\
\hline 政策制度类 & KP, 巴黎协定, CDM, REDD+, 部门, 响应机制 & 21 & 制定综合评估框架和决策支持系统 ${ }^{[60]}$, 比较分析法 \\
\hline 经济环保类 & $\begin{array}{l}\text { 减缓气候变化, 碳汇, 成本, 价格, 预算, 信贷(大气碳、 } \\
\text { 生物量、土壤有机碳、生物多样性) }\end{array}$ & 46 & $\begin{array}{l}\text { 空间成本效益分析法 }{ }^{[60]} \text {, 层次分析法, 土地利用和资源 } \\
\text { 配置建模系统 }{ }^{[18]}, \text { 一般均衡模型 }\end{array}$ \\
\hline
\end{tabular}

表4 涉及 LULUCF 林业碳问题的国内外代表期刊及研究对比

Table 4 Comparison of representative periodicals and research on LULUCF in China and internationally

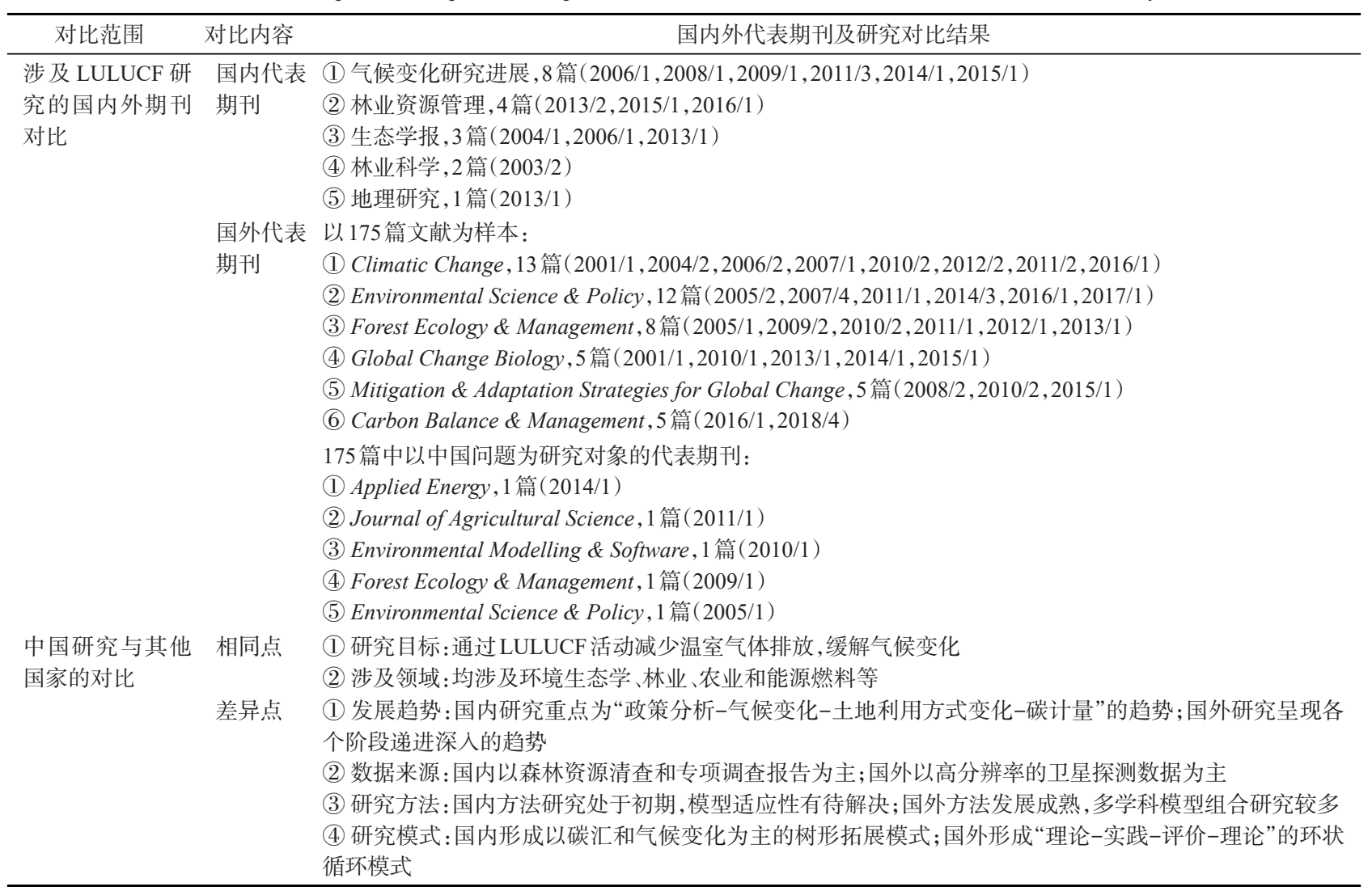

注:括号内数字为年份/篇数,如 $2006 / 1$ 即该期刊 2006 年发表 1 篇相关文献。 
表以《气候变化研究进展》和《生态学报》为主, 另外 还涉及了环境、林业、农业经济和生物学等期刊, 虽 然国内 LULUCF 研究起步较晚, 但各领域对该问题 的研究热点不断关注。以中国 LULUCF 林业碳问 题为研究对象的研究成果主要发表在Journal of $\mathrm{Ag}$ ricultural Science 和 Forest Ecology \& Management 等期刊, 以土地管理决策对碳固存的影响和生物量 扩展因子的研究为主, 这说明了关于中国 LULUCF 林业碳问题的研究受到了国际主流刊物的重视。

比较研究内容发现, 国内外对 LULUCF 林业碳 问题研究的目标和涉及领域是一致的, 但在发展趋 势、研究方法、数据来源和研究模式方面存在差 异。(1)从发展趋势看, 国内各个时期研究各有侧重 点, 具体为“政策分析-气候变化-土地利用方式变 化一碳计量”的变化趋势; 国外研究呈现各个阶段递 进深人的趋势, 从最初的碳固存和成本问题到 LULUCF 活动及影响研究, 重视发展中国家减排潜力 和森林管理, 最后呈现各领域综合研究的趋势。(2) 在数据获取方面也存在差距, 国内以森林资源清查 和专项调查报告等为主要来源, 国外更多使用卫星 探测数据, 分辨率显著提高。(3)在研究方法方面, 国内仍处于研究初期, 需要考虑借鉴其他国家模型 方法的适应性及改进问题, 国外对 LULUCF 的研究 方法相对成熟, 以比较研究和多学科模型组合为 主。如针对重大林业管理项目诸如退耕还林、天然 林保护工程等, 国内倾向于使用定性分析法评价其 实施效果和影响, 国外的研究侧重于借助模型预测 土地利用可能出现的变化和对碳活动造成的影响, 更具针对性和说服力。(4)在研究模式上, 国内关注 生物量、碳储量、HWP、森林管理和森林碳汇等方 面, 这些研究彼此相对独立, 并侧重对碳汇核算和 气候变化影响的研究, 形成以碳汇和再造林为主的 树形拓展模式; 国外从聚焦于 UNFCCC 和 KP 中关 于LULUCF 的理论分析, 到基于实践的具体样本研 究, 发展为构建各种评价模型, 进行评价提炼后再 对理论进行改进, 形成 “理论-实践-评价-理论” 的 环状循环模式。与其他国家研究对比, 中国未来应 在研究方法的改进和数据获取、林业管理项目评价 方法和整合不同研究成果并付诸实践等方面加大 研究, 以实现减排目标为减缓气候变化作出贡献。

\section{4 对部分LULUCF研究问题的讨论}

\section{1 碳活动核算的主流方法学}

研究碳活动有利于了解不同土地利用变化对 碳循环、温室气体排放及气候变化的影响, 为制定 合理减少碳排放政策提供借鉴。当前碳活动研究 方法学主要涉及单一模型法和组合研究法, 其中单 一模型法以簿记模型为代表, 组合研究法则以比较 研究和多学科模型整合为主。

根据研究区域的土地变化类型、经济发展水 平、能源消耗模式等进行仿真, 可以有效准确地预 测大时间尺度(10 100 年)和大空间尺度(城市区域 一全球区域) 的未来碳储量变化。簿记模型是研究 LULUCF 林业碳问题中使用最多的方法 ${ }^{[3238,841]}$, 该模 型是核算陆地生态系统和大气碳素交换过程的碳 收支模型, 用于研究土地利用变化引起的碳排放和 碳吸收。通过簿记模型跟踪单位土地采伐或重新 造林的活植被、枯死植物、木制品和土壤中的碳, 可 以估计研究区域碳密度和碳储量, 或结合碳储量变 化值评估生态系统碳储量和碳净排放量。

当单一的模型工具不能解决 LULUCF 引起的 碳量变化及对经济环境等的影响问题时, 可以将模 型工具与其他方法结合, 组合研究法就成为当前最 主流的研究选择,组合研究法使用中主要有以下几 种处理方法。(1) 比较不同方法的结果 ${ }^{[32,49]}$ 。碳核算 方法在数据处理、情景假设或时间跨度上都可能存 在限制, 对比并分析 2 种方法可以互相弥补缺点, 减 小误差。当研究的碳量结果与评估报告或政策制 度预期达到的减排贡献不符时, 则需要更精确的数 据和案例作出证明, 为改善减排政策制度提供依

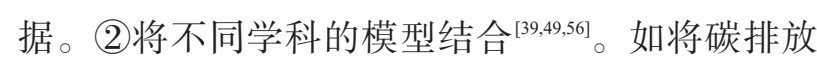
核算和经济评估结合可以估计森林未来碳储量变 化对碳价格的影响, 土地利用模型和能源模型结合 可以评估 LULUCF 部门和能源供应部门之间的权 衡和制约关系, 全球粮食预测模型和森林管理模型 结合可以用粮食供求情景分析森林未来生长情况 和对碳排放或清除的影响。(3)从数据来源和规划 工具等方面提高精确度 ${ }^{[37,4,753]}$ 。使用卫星探测数据 是提高精确度的重要方法, 有利于提高记录森林面 积变化、测量生物量密度变化和估计森林覆盖率的 空间分辨率, 对分析土地利用变化引起的碳活动具 
有重要作用。路线图规划方法简洁直观, 能突出重 点环节, 用路线图展现减排措施能清晰地表示出碳 减排关键部门和步骤。为了确保一致性, 不同模型 使用的数据应尽可能地统一, GIS 覆盖图可以使模 型和数据库之间的面积标准化,避免两者因分类差 异导致的土地利用变化的误差。

\section{2 林业管理项目的碳减排潜力}

通过实施林业管理项目, LULUCF 活动可以利 用保护现有的森林碳库和增加碳储量 2 种方式缓解 气候变化。林业管理项目是为了合理有效地管理 森林, 最大限度开发并发挥出森林提供生态服务和 林产品等用途的措施。充分设计和实施林业管理 项目 ${ }^{[42,43,45]}$, 如改善耕地和牧地管理、恢复退化的土 地、对减少砍伐予以补偿、权衡环境保护与其他森 林产品和服务等, 可以降低森林破坏程度, 恢复森 林覆盖率和森林密度, 从而增加碳储量, 为缓解气 候变化作出贡献。

评估林业管理项目碳减排潜力的方法大致分 为 2 类: 评估法和情景预测法。评估法用于评估政 策对减缓气候变化的贡献、比较不同管理过程的重 要程度、评价管理行为变化的潜在影响等。使用评 估法时,在确定碳减排影响因素和准则上存在差 异, 林业管理项目对环境和林农的影响等变量也难 以量化, 这些不确定性和主观性都会使评估结果缺 乏可信度。情景预测法可以结合现有的数据和林 业管理项目发展趋势, 借助模型预测土地利用可能 出现的变化和对碳活动造成的影响 ${ }^{[42,52,56]}$, 模型包括 IMAGE 、IMPACT 和 GLOBIOM 等。基于已有数据 和土地变化趋势估计未来土地利用变化对碳排放 产生的影响, 进而对气候变化趋势进行预测, 可以 针对性地提出改善土地利用管理的方法, 提高社会 对气候变化的关注度并大力实施促进碳减排的林 业管理项目。

\section{3 碳预算的紧迫性和碳抵消面临的争议}

碳预算是规定在给定时间内允许排放到大气 中碳的数目。巴黎协定警示全球需要进人平衡碳 预算的新世界 ${ }^{[33]}$ 。影响制定碳预算的因素包括预算 类型、基本数据和模式、情景选择、温度响应的时间 尺度、 $\mathrm{CO}_{2}$ 排放和非 $\mathrm{CO}_{2}$ 温室气体排放的增暖贡献 和适用性等方面 ${ }^{[0,61]}$ 。明确的森林面积变化信息、
土壤变化和气候变化等影响因素不易测定,会导致 制定碳预算目标和为各部门分配碳预算过程的不 确定性 ${ }^{[2]}$ 。碳预算目标的制定及影响因素的测定是 平衡碳预算的关键, 如何降低碳预算的不确定性, 并根据碳预算制定或调整减排方案具有重要现实 意义。在 LULUCF 活动碳预算方案的设计中, 关键 是 LULUCF 部门在减排量、减排方式和减排效益三 者之间的权衡。通过将 LULUCF 活动的碳排放、碳 减排和碳排放交易等活动以预算的形式加以系统 规划, 形成具体的预算任务分解到各部门,借助路 线图合理规划执行过程 ${ }^{[33]}$,并在执行过程中进行实 时控制和调整, 以实现 LULUCF 活动碳减排目标, 并核算相应的减排成本和收益,确定减排效益最优 的减排方案。

碳抵消机制旨在促进个人和企业为环保事业 作出贡献。个人和企业可以通过提供费用参与植 树造林或其他改善环境的项目,抵消其产生的温室 气体。Brown 等 ${ }^{[63}$ 概括了可作为潜在碳抵消项目的 林业管理类型,并提出了无具体碳抵消项目实施时 估计碳储量变化的计算方法。然而,碳抵消机制是 否可以真正起到实质减排作用仍存在争议。Ander$\operatorname{son}^{[35]}$ 对碳抵消的合理性提出疑问,认为碳抵消没有 科学合理性,具有危险的误导性,会导致全球排放 量增长。Hyams 等认为碳抵消比真正缓解气候变 化的减排机制更能满足 “碳信用额度”, 即规定付出 费用就可以排放同等额度的碳 ${ }^{[4,65]}$ 。理论上碳抵消 机制似乎能够达到“碳中和”目标, 但这很容易给企 业造成“只要提供足够费用就可以任意排放温室气 体”的错误导向。在实施植树造林等项目时,工作 人员因交通和饮食会增加碳排放,个人和企业也会 因已经提供费用的心理而增加更多碳排放,这些都 会导致碳排放总量增加。这些因项目实施而增加 的碳排放如何影响环境,暂无确定性方法量化。同 时项目的实施效果会受森林面积变化、植被生长周 期和气候变化等因素的影响。因此植树造林等项 目中碳排放与碳吸收共存, 增加碳排放总量, 不能 确保个人和企业排放的碳被完全吸收并封存。总 之, 在不能保证减少实质排放的情况下, 碳抵消机 制本质上与 “先污染后治理” 的做法相同, 为实现 “碳中和”而推进的碳抵消机制仍需要长期观测和 
科学数据的支持。

\section{5 结论与展望}

本文借助 CiteSpace 对 LULUCF 科研进展进行 了分析, 通过文献梳理和数据挖掘, 归纳了 LULUCF 在林业碳领域的研究重点和研究趋势。研究 表明: 1)1999-2018 年, LULUCF 林业碳问题的研 究主题重点集中于 3 个类群 6 个聚类。其中 3 个类 群问题可总结为 “制度和方法的改进” “林业管理行 为”和 “环境目标”; 6 个聚类问题涉及: “多源比较” “林业发展项目” “其他土地利用” “十大短期部门基 准”“信贷核算”和“碳”。(2)德国、意大利、英国和美 国是研究 LULUCF 的主要国家, 研究分别侧重于毁 林、林业项目、经济成本问题和部门响应机制。荷 兰、澳大利亚和瑞典注重减排机制和生物多样性的 权衡, 芬兰和奥地利注重森林树种的研究。(3LULUCF 林业碳科学在社会科学方面的重点关注问题 是管理类、政策制度类和经济环保类,主要方法是 工程管理学和经济学方法, 涉及情景分析法、层次 分析法、一般均衡模型和空间成本效益分析。

中国目前关于 LULUCF 的研究侧重于生态系 统碳库、温室气体清单编制及碳汇计量监测体系等 问题, 结合国际间 LULUCF 林业碳科学研究进展和 趋势,本文建议中国未来应对以下问题给予关注:

(1) 对碳排放、碳吸收和碳储存等碳活动的碳 量核算, 有利于了解不同 LULUCF 活动对碳循环、 温室气体排放及气候变化的影响, 为制定合理减少 碳排放政策提供借鉴。单一模型法中使用最多的 是簿记模型, 组合研究法则以比较研究和多学科模 型整合为主。LULUCF 本质上是因土地利用及结 构变动引致的林业碳问题, 因此关于土地利用及变 动的基础科学是评估林业碳问题的基础, 提高卫星 探测数据和 GIS 覆盖图的使用率有利于降低误差, 提高分析土地利用变化的精度和空间分辨能力。

(2)依据林业管理项目, LULUCF 活动可以从 保护现有的森林碳库和增加碳储量 2 个方面缓解气 候变化。利用簿记模型、全球环境综合评估模型和 全球生物圈管理模式等方法预测林业管理项目的 碳减排潜力,这对制定及调整响应气候变化的林业 政策有重要意义。中国下一步的研究和实践应充 分重视重大林业管理项目的气候功能评价和风险
点防控,如科学评估重要林区在应对气候变化中的 减排作用、改善易着火森林的管理和保护、权衡森 林生态服务系统的碳价值、通过改善耕地和牧地管 理提高退化土地的林业利用等。

(3) 控制碳活动关联的碳抵消、碳预算等对减 少碳排放问题的政策设计具有重要借鉴价值。碳 预算目标的制定及影响因素的测定是平衡碳预算 的关键, 如何降低碳预算的不确定性, 并根据碳预 算制定或调整减排方案具有重要现实意义。需注 意碳抵消机制与实质性减排机制的实际效果比较, 有限度的试行碳抵消,科学评估其实现“碳中和”的 能力及贡献。中国需要加快将 LULUCF 的碳排放 以预算的形式加以系统规划,形成具体的预算任务 分解到土地利用各部门,核算相应的减排成本和收 益, 确定减排效益最优的减排方案, 以实现平衡碳 预算的目标。

\section{参考文献(References)：}

[1] 张小全, 陈先刚, 武曙红. 土地利用变化和林业活动碳它量变化 测定与监测中的方法学问题[J]. 生态学报, 2004, 24(9): 20682073. [Zhang X Q, Chen X G, Wu S H. Methodological issues related to measuring and monitoring carbon stock changes induced by land use change and forestry activities[J]. Acta Ecologica Sinica, 2004, 24(9): 2068-2073.]

[2] 白彦锋, 姜春前, 张守攻, 等. 气候变化谈判中木质林产品的相 关概念及其碳储量核算[J]. 林业科学, 2011, 47(1): 158-164. [Bai Y F, Jiang C Q, Zhang S G, et al. Definitions and carbon stocks accounting approaches of harvested wood products in climate change negotiation[J]. Scientia Silvae Sinicae, 2011, 47(1): 158-164.]

[3] Watson R T, Noble I, Bolin B, et al. Land Use, Land-Use Change, and Forestry: A Special Report of the IPCC[M]. Cambridge: Cambridge University Press, 2000.

[4] IPCC. Climate Change 1995: The Science of Climate Change[M]. Cambridge: Cambridge University Press, 1996.

[ 5 ] IPCC. Climate Change 2001: The Scientific Basis[M]. Cambridge: Cambridge University Press, 2001.

[ 6] IPCC. Climate Change 2007: The Physical Science Basis[M]. Cambridge: Cambridge University Press, 2007.

[ 7 ] IPCC. Climate Change 2013: The Physical Science Basis[M]. Cambridge: Cambridge University Press, 2013.

[ 8 ] Bernoux M, Carvalho M C S, Volkoff B, et al. $\mathrm{CO}_{2}$ emission from mineral soils following land- cover change in Brazil[J]. Global 
Change Biology, 2001, 7(7): 779-787.

[9] Gundimeda H. How "sustainable" is the "sustainable development objective" of CDM in developing countries like India?[J]. Forest Policy and Economics, 2004, 6(3-4): 329-343.

[10] Garcia-Quijano J F, Deckmyn G, Moons E, et al. An integrated decision support framework for the prediction and evaluation of efficiency, environmental impact and total social cost of domestic and international forestry projects for greenhouse gas mitigation: Description and case studies[J]. Forest Ecology and Management, 2005, 207(1-2): 245-262.

[11] Cienciala E, Tomppo E, Snorrason A, et al. Preparing emission reporting from forests: Use of national forest inventories in European countries[J]. Silva Fennica, 2008, 42(1): 73-88.

[12] Federici S, Vitullo M, Tulipano S, et al. An approach to estimate carbon stocks change in forest carbon pools under the UNFCCC: The Italian case[J]. Iforest Biogeosciences and Forestry, 2008, 21 (1): 86-95.

[13] Blujdea V, Bird D N, Robledo C. Consistency and comparability of estimation and accounting of removal by sinks in afforestation/reforestation activities[J]. Mitigation and Adaptation Strategies for Global Change, 2010, 15(1): 1-18.

[14] Latta G S, Baker J S, Ohrel S. A Land Use and Resource Allocation (LURA) modeling system for projecting localized forest $\mathrm{CO}_{2}$ effects of alternative macroeconomic futures[J]. Forest Policy and Economics, 2018, 87: 35-48.

[15] Olsson A, Gronkvist S, Lind M, et al. The elephant in the room: A comparative study of uncertainties in carbon offsets[J]. Environmental Science and Policy, 2016, 56: 32-38.

[16] Pradhan B B, Shrestha R M, Hoa N T, et al. Carbon prices and greenhouse gases abatement from agriculture, forestry and land use in Nepal[J]. Global Environmental Change-Human and Policy Dimensions, 2017, 43: 26-36.

[17] Lintunen J, Laturi J, Uusivuori J. How should a forest carbon rent policy be implemented?[J]. Forest Policy and Economics, 2016, 69: 31-39.

[18] Vauhkonen J, Packalen T. Uncertainties related to climate change and forest management with implications on climate regulation in Finland[J]. Ecosystem Services, 2018, 33: 213-224.

[19] 张小全. LULUCF 在《京都议定书》履约中的作用[J]. 气候变化 研究进展, 2011, 7(5): 369-377. [Zhang X Q. Roles of LULUCF activities in Kyoto Protocol compliance[J]. Climate Change Research, 2011, 7(5): 369-377.]

[20] 张小全, 侯振宏. 第二承诺期 LULUCF 有关议题谈判进展与对 策建议[J]. 气候变化研究进展, 2009, 5(2): 95-102. [Zhang X Q, Hou Z H. Progress on LULUCF negotiations for the second commitment period[J]. Climate Change Research, 2009, 5(2): 95-102.]

[21] 张小全. 土地利用变化和林业清单方法学进展 [J]. 气候变化研 究进展, 2006, 2(6): 265-268. [Zhang X Q. Progress in methodolo- gy of land use change and forestry inventory[J]. Climate Change Research, 2006, 2(6): 265-268.]

[22] 刘硕, 李玉娥, 高清竹, 等. 不同减排领域对附件 B 缔约方完成 《京都议定书》第一承诺期减排目标的贡献[J]. 气候变化研究 进展, 2015, 11(2): 131-137. [Liu S, Li Y E, Gao Q Z, et al. Contribution of different emission reduction areas to Annex B Parties' achievement of emission reduction targets in the first commitment period of Kyoto Protocol[J]. Climate Change Research, 2015, 11 (2): 131-137.]

[23] 刘硕, 李玉娥, 高清竹, 等. 后京都时期 LULUCF 潜在核算规则 分析[J]. 气候变化研究进展, 2011, 7(4): 294-300. [Liu S, Li Y E, Gao Q Z, et al. Analysis of LULUCF accounting rules for PostKyoto[J]. Climate Change Research, 2011, 7(4): 294-300.]

[24] 李玉娥, 秦晓波, 万运帆, 等. 第二承诺期土地利用、土地利用变 化与林业规则的各方观点及对策建议 [J]. 气候变化研究进展, 2008, 4(5): 277-281. [Li Y E, Qin X B, Wan Y F, et al. Parties views and suggestions for land use, land-use change and forestry rules for the second commitment period[J]. Climate Change Research, 2008, 4(5): 277-281.]

[25] 赵志平, 邵全琴, 王军邦, 等. 中国 LULUCF 碳核算与决策支持 系统设计与开发 [J]. 地球信息科学学报, 2010, 12(6): 743-752. [Zhan Z P, Shao Q Q, Wang J B, et al. Design and development of the carbon accounting and decision support system of LULUCF in China[J]. Journal of Geo-information Science, 2010, 12(6): 743752.]

[26] 林德荣, 李智勇. 减少毁林和森林退化引起的排放: 一个综述视 角的分析[J]. 世界林业研究, 2010, 23(2): 1-4. [Lin D R, Li Z Y. Reducing emissions from deforestation and forest degradation: An analysis from overview perspective[J]. World Forestry Research, 2010, 23(2): 1-4.]

[27] 董玮, 田淑英, 刘浩. 林业生态经济发展多维度公共政策选择与 测度[J]. 中国人口・资源与环境, 2017, 27(11): 149-158. [Dong W, Tian S Y, Liu H. Choices of public policies for forestry ecological economy development and the multi-dimensional measurements[J]. China Population, Resources and Environment, 2017, 27 (11): 149-158.]

[28] 侯剑华, 胡志刚. CiteSpace 软件应用研究的回顾与展望[J]. 现 代情报, 2013, 33(4): 99-103. [Hou J H, Hu Z G. Review on the application of CiteSpace at home and abroad[J]. Journal of Modern Information, 2013, 33(4): 99-103.]

[29] 肖明, 邱小花, 黄界, 等. 知识图谱工具比较研究 $[J]$. 图书馆杂 志, 2013, 32(3): 61-69. [Xiao M, Qiu X H, Huang J, et al. Comparative study on knowledge mapping tools[J]. Library Journal, 2013, 32(3): 61-69.]

[30] 赵丹群. 基于 CiteSpace 的科学知识图谱绘制若干问题探讨 [J]. 情报理论与实践, 2012, 35(10): 56-58. [Zhao D Q. Probe into several problems relating to mapping knowledge domains based on CiteSpace[J]. Information Studies: Theory and Application, 2012, 
35(10): 56-58.]

[31] 陈悦, 陈超美, 胡志刚, 等. 引文空间分析原理与应用 $[M]$. 北京: 科学出版社, 2015. [Chen Y, Chen C M, Hu Z G, et al. Principle and Application of Citation Space Analysis[M]. Beijing: Science Press, 2015.]

[32] Baccini A, Goetz S J, Walker W S, et al. Estimated carbon dioxide emissions from tropical deforestation improved by carbon-density maps[J]. Nature Climate Change, 2012, 2(3): 182-185.

[33] Anderson K. Duality in climate science[J]. Nature Geoscience, 2015, 8(12): 898-900.

[34] Brando P M, Balch J K, Nepstad D C, et al. Abrupt increases in Amazonian tree mortality due to drought-fire interactions[J]. Proceedings of the National Academy of Sciences of the United States of America, 2014, 111(17): 6347-6352.

[35] Anderson K. The inconvenient truth of carbon offsets[J]. Nature, 2012, 484(7392): 7-7.

[36] Brienen R J W, Phillips O L, Feldpausch T R, et al. Long-term decline of the Amazon carbon sink[J]. Nature, 2015, 519(7543): 344348.

[37] Houghton R A. How well do we know the flux of $\mathrm{CO}_{2}$ from landuse change?[J]. Tellus Series B-Chemical and Physical Meteorology, 2010, 62(5): 337-351.

[38] Harris N L, Brown S, Hagen S C, et al. Baseline map of carbon emissions from deforestation in tropical regions[J]. Science, 2012, 336(6088): 1573-1576.

[39] Saatchi S S, Harris N L, Brown S, et al. Benchmark map of forest carbon stocks in tropical regions across three continents[J]. Proceedings of the National Academy of Sciences of the United States of America, 2011, 108(24): 9899-9904.

[40] Pan Y D, Birdsey R A, Fang J Y, et al. A large and persistent carbon sink in the world's forests[J]. Science, 2011, 333(6045): 988993.

[41] Houghton R A, House J I, Pongratz J, et al. Carbon emissions from land use and land-cover change[J]. Biogeosciences, 2012, 9(12): $5125-5142$

[42] Guo L B, Gifford R M. Soil carbon stocks and land use change: A Meta analysis[J]. Global Change Biology, 2002, 8(4): 345-360.

[43] Smith P, Martino D, Cai Z C, et al. Greenhouse gas mitigation in agriculture[J]. Philosophical Transactions of the Royal Society BBiological Sciences, 2008, 363(1492): 789-813.

[44] Smith P, Ashmore M R, Black H I J, et al. The role of ecosystems and their management in regulating climate, and soil, water and air quality[J]. Journal of Applied Ecology, 2013, 50(4): 812-829.

[45] Ellison D, Lundblad M, Petersson H. Carbon accounting and the climate politics of forestry[J]. Environmental Science and Policy, 2011, 14(8): 1062-1078.

[46] Smith P, Haberl H, Popp A, et al. How much land-based greenhouse gas mitigation can be achieved without compromising food security and environmental goals?[J]. Global Change Biology, 2013, 19(8): 2285-2302

[47] Hansen M C, Potapov P V, Moore R, et al. High-resolution global maps of 21st-century forest cover change[J]. Science, 2013, 342 (6160): 850-853.

[48] Rogelj J, Elzen M, Höhne N, et al. Paris agreement climate proposals need a boost to keep warming well below $2^{\circ} \mathrm{C}[\mathrm{J}]$. Nature, 2016 , 534(7609): 631-639.

[49] Springmann M, Godfray H C J, Rayner M, et al. Analysis and valuation of the health and climate change cobenefits of dietary change [J]. Proceedings of the National Academy of Sciences of the United States of America, 2016, 113(15): 4146-4151.

[50] Bajzelj B, Richards K S, Allwood J M, et al. Importance of fooddemand management for climate mitigation[J]. Nature Climate Change, 2014, 4(10): 924-929.

[51] Herrero M, Henderson B, Havlík P, et al. Greenhouse gas mitigation potentials in the livestock sector $[J]$. Nature Climate Change, 2016, 6(5): 452-461.

[52] Frank S, Havlık P, Soussana J F, et al. Reducing greenhouse gas emissions in agriculture without compromising food security[J]. Environmental Research Letters, 2017, DOI: 10.1088/1748-9326/ aa8c83.

[53] Rockstrom J, Gaffney O, Rogelj J, et al. A roadmap for rapid decarbonization[J]. Science, 2017, 355(6331): 1269-1271.

[54] Grassi G, Elzen M G J, Hof A F, et al. The role of the land use, land use change and forestry sector in achieving Annex I reduction pledges[J]. Climatic Change, 2012, 115(3-4): 873-881.

[55] Nabuurs G J, Lindner M, Verkerk P J, et al. First signs of carbon sink saturation in European forest biomass[J]. Nature Climate Change, 2013, 3(9): 792-796.

[56] Bottcher H, Verkerk P J, Gusti M, et al. Projection of the future $\mathrm{EU}$ forest $\mathrm{CO}_{2}$ sink as affected by recent bioenergy policies using two advanced forest management models[J]. Global Change Biology Bioenergy, 2012, 4(6): 773-783.

[57] Grassi G, House J, Dentener F, et al. The key role of forests in meeting climate targets requires science for credible mitigation[J]. Nature Climate Change, 2017, 7(3): 220-226.

[58] Griscom B W, Adams J, Ellis P W, et al. Natural climate solution $[J]$. Proceedings of the National Academy of Sciences of the United States of America, 2017, 114(44): 11645-11650.

[59] Minang P A, Bressers H T A, Skutsch M M, et al. National forest policy as a platform for biosphere carbon management: The case of community forestry in Cameroon[J]. Environmental Science and Policy, 2007, 10(3): 204-218.

[60] Wang J, Chen J M, Ju W M, et al. IA-SDSS: A GIS-based land use decision support system with consideration of carbon sequestration[J]. Environmental Modelling and Software, 2010, 25(4): 539-553. 
[61] Karjalainen T, Pussinen A, Liski J, et al. Scenario analysis of the impacts of forest management and climate change on the European forest sector carbon budget[J]. Forest Policy and Economics, 2003, 5(2): 141-155.

[62] DeFries R S, Houghton R A, Hansen M C, et al. Carbon emissions from tropical deforestation and regrowth based on satellite observations for the 1980s and 1990s[J]. Proceedings of the National Academy of Sciences of the United States of America, 2002, 99 (22): $14256-14261$.
[63] Brown S, Burnham M, Dellaney M, et al. Issues and challenges for forest-based carbon- offset projects: A case study of the Noel Kempff climate action project in Bolivia[J]. Mitigation and Adaptation Strategies for Global Change, 2000, 5(1): 99-121.

[64] Hyams K, Fawcett T. The ethics of carbon offsetting[J]. Wiley Interdisciplinary Reviews-Climate Change, 2013, 4(2): 91-98.

[65] Subak S. Forest protection and reforestation in Costa Rica: Evaluation of a clean development mechanism prototype[J]. Environmental Management, 2000, 26(3): 283-297.

\title{
Research progress of forestry carbon issues related to land use, land-use change and forestry
}

\author{
XU Enyin ${ }^{1,2}$, TAO Yun ${ }^{1,2}$, YANG Hongqiang ${ }^{1,2,3}$ \\ (1. College of Economics and Management, Nanjing Forestry University, Nanjing 210037, China; \\ 2. Research Center for Economics and Trade in Forest Products, The State Forestry Administration, Nanjing 210037, China; \\ 3. Center for the Yangtze River Delta's Socioeconomic Development, Nanjing University, Nanjing 210093, China)
}

\begin{abstract}
The carbon issues associated with land use, land-use change and forestry (LULUCF) is an important issue affecting adaptation and mitigation of climate change. Clarifying the international research progress related to forestry and carbon issues in the past 20 years is of great significance for the formulation and adjustment of forestry policies in response to climate change. This article analyzes 175 documents in the Web of Science database from 1999 to 2018 and summarizes the research trends of LULUCF carbon research with the CiteSpace tool. The results show that: (1) The current research topics of LULUCF are "improvement of systems and methods," "forest management behavior," and "environmental objectives." (2) Germany, Italy, the United Kingdom, and the United States are the main countries studying LULUCF. They focus on deforestation, forestry projects, economic issues, and sectoral response mechanisms respectively. (3) The focuses of social science research on LULUCF are land use management, response mechanism of policies, and economic problems. The main methods are engineering management and economics approaches represented by scenario analysis and general equilibrium models. This article provides some relevant suggestions for future research on LULUCF: improving LULUCF carbon accounting methods and the theory and means of monitoring land use and dynamic changes; paying attention to the scientific evaluation of forest management projects and its influence on the adjustment of forestry policies; and accelerating LULUCF carbon budget research and the formulation of emission reduction plans by land use sectors.
\end{abstract}

Key words: LULUCF; CiteSpace; forestry carbon; cluster; forest management projects; carbon budget; carbon offset 